\title{
Adsorption of Amyloid Beta Peptide by Metal-Organic Frameworks
}

\author{
Zachary L. Mensinger,* Brenna L. Cook, and Elsie L. Wilson
}

Cite This: ACS Omega 2020, 5, 32969-32974

Read Online

ABSTRACT: Metal-organic frameworks (MOFs) are capable of adsorbing a wide range of molecules. In addition to the more commonly investigated small molecules, researchers have demonstrated that MOFs adsorb much larger molecules, such as proteins and peptides. We have investigated whether MOFs are capable of adsorbing amyloid beta peptide. Amyloid beta plays a pivotal role in the progression of Alzheimer's disease because individual copies of the peptides can aggregate, forming neurotoxic oligomers and

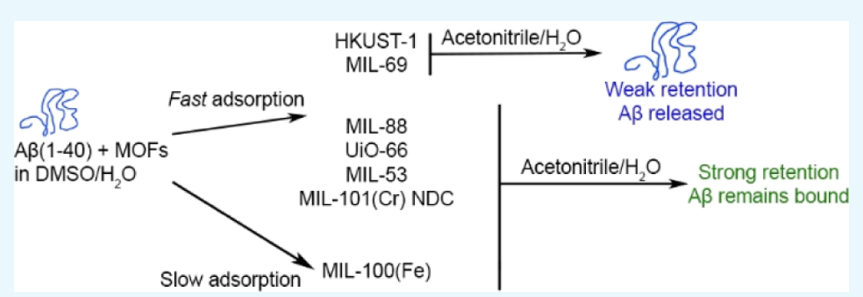
the amyloid plaques found in brains of Alzheimer's patients. After synthesizing a number of commonly studied MOFs, their adsorption capabilities were tested. We found that the MOFs tested readily adsorbed small amounts of amyloid beta (as determined by gel electrophoresis). It was determined that in most cases, adsorption occurs rapidly, with complete adsorption within minutes of incubation. Overall adsorption capacity was found to vary between different MOFs as well. Once adsorbed, amyloid beta peptide can subsequently be eluted from some MOFs by treatment with acetonitrile/water solutions, though retention strength varied between different MOFs. In some cases, MOFs that showed complete adsorption also saw high levels of peptide elution, but others showed little to no elution of the peptide. Together these data can help us begin to understand the interactions between amyloid beta and MOFs.

\section{INTRODUCTION}

Alzheimer's disease $(\mathrm{AD})$ is a tragic disease with a complex etiology. Though a direct, singular cause of $\mathrm{AD}$ remains elusive and might not exist, there is no doubt that amyloid beta $(\mathrm{A} \beta)$ plays a role in the progression of the disease, as the precursor for amyloid plaques. ${ }^{1} \mathrm{~A} \beta$ readily aggregates, first forming small oligomers, then larger protofibrils and fibrils, and finally plaques. $\mathrm{A} \beta$ aggregation is a dynamic process, making it hard to characterize the impacts of just one species that exists as part of a complex mixture. Even if a particular aggregate species is separated through methods such as size-exclusion chromatography, it could continue to aggregate or disaggregate after separation. In light of these challenges, techniques to isolate particular $\mathrm{A} \beta$ species would be valuable. Metal-organic frameworks (MOFs), which offer a range of pore sizes and properties that can be tailored based on the metal ions and ligands that are chosen for their synthesis, might have the potential to offer selective adsorption activity. ${ }^{2}$ MOFs have previously been shown to adsorb a number of different peptides and proteins, with many reports in particular about their ability to incorporate enzymes. ${ }^{3}$ Fewer reports have examined the interactions between MOFs and $\mathrm{A} \beta$, mostly in studies focused on the potential of MOF-containing systems to attenuate $^{\text {aggregation }}{ }^{4 a, b}$ or detect the peptide. ${ }^{4 c-f}$ These studies have generally utilized MOFs as part of a more complex experimental system, and we have not encountered studies testing whether $\mathrm{A} \beta$ and MOFs alone directly interact. Therefore, in this study, we sought to establish whether $\mathrm{A} \beta$ could be adsorbed by MOFs. Several MOFs were synthesized and then incubated with solutions of monomerized $\mathrm{A} \beta(1-$ 40). ${ }^{5,6}$ MOFs were screened for adsorption capability, rate, relative strength of retention, and adsorption capacity. It was determined that while each MOF tested adsorbs $\mathrm{A} \beta$, the rate, capacity, and strength of retention vary.

\section{RESULTS}

Our initial studies examined whether MOFs were capable of adsorbing $\mathrm{A} \beta(1-40)$. After $2 \mathrm{~h}$ of incubation, supernatants from samples incubated with all MOFs in this study were below fluorimetric detection limits. Results from silver staining generally agree with those measured using fluorimetry, though they provided a higher level of sensitivity (Figure 1a-c). In this case, all MOFs other than MIL-100(Fe) showed full adsorption of $\mathrm{A} \beta$ from solution. For MIL-100(Fe), about $12 \%$ of the initial amount of peptide remained after the first incubation. Though fluorimetric analysis failed to detect peptide in the MIL-100(Fe) sample, this is likely due to concentrations being below detection limits of the instrument. Testing the concentrations after a first MIL-100(Fe) incubation with bicinchoninic acid (BCA) assay supported

Received: August 19, 2020

Accepted: December 3, 2020

Published: December 14, 2020 


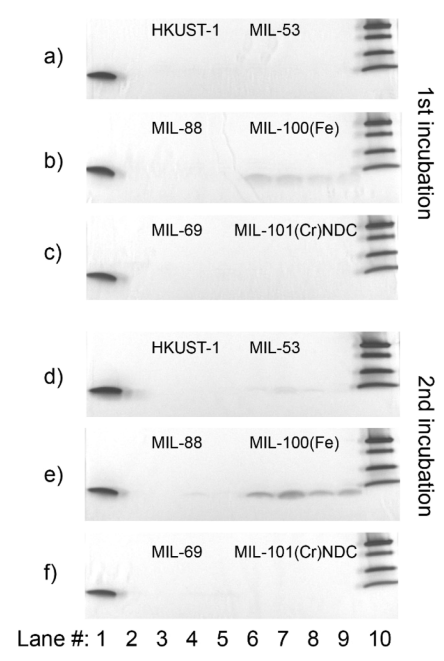

Figure 1. $(a-f)$ Gel electrophoresis results of representative incubations with MOFs and $A \beta$. $(a-c)$ First incubation and $(d-f)$ second incubation with the same MOF samples. On each gel, lane 1 is the control/initial and lane 10 is the reference ladder (Bio-Rad Precision Plus, bands at 10, 15, 20, and $25 \mathrm{kD}$ shown). Lanes $2-5$ and 6-9 represent quadruplicate incubations with the same MOF, respectively. (a,d) HKUST-1 and MIL-53, (b,e) MIL-88B and MIL$100(\mathrm{Fe})$, and (c,f) MIL-69 and MIL-101(Cr) NDC. Lanes 6-9 of the gels in (b,e) show MIL-100(Fe), the only MOF with incomplete adsorption over this timeframe.

the values of peptides remaining detected by gel electrophoresis.

The same samples were subjected to a second incubation to test whether additional peptide would be adsorbed. When analyzed by fluorimetry, the results were largely consistent with the first incubation. The initial $\mathrm{A} \beta$ concentrations were similar, around $50 \mu \mathrm{g} / \mathrm{mL}$. All samples were still below fluorimetric detection limits after incubation, so silver staining was again primarily used (Figure $1 \mathrm{~d}-\mathrm{f})$. MIL-100 $(\mathrm{Fe})$ again was the only sample with significant amounts of $\mathrm{A} \beta$ left in solution (Figure 1e). This time, about $32 \%$ of the $A \beta$ remained in solution after incubation, and all others showed less than $3 \%$ of the original amount remaining. These results again support that at least among the MOFs tested, most of them readily adsorb $\mathrm{A} \beta$, but as more $\mathrm{A} \beta$ is introduced, we likely start to see a saturation point, evidenced by more unadsorbed $\mathrm{A} \beta$ remaining in the second MIL-100(Fe) incubation.

It was next tested whether $\mathrm{A} \beta$ could be desorbed or eluted from the MOF solids. This was intended to determine whether (1) $\mathrm{A} \beta$ remained intact after the incubation and was not hydrolyzed or otherwise degraded, (2) the peptide formed stronger interactions with some MOFs than others, and (3) peptides were not simply aggregating and precipitating with the MOF. Rinsing the MOF solids with $\mathrm{H}_{2} \mathrm{O}$ alone showed no significant presence of $\mathrm{A} \beta$, suggesting that water does not elute or wash off the peptide from the MOF-this has previously been suggested as evidence for pore, as opposed to surface, adsorption. $^{3 \mathrm{c}}$ The control peptide level for desorption corresponds to only a single incubation equivalent, so roughly $200 \%$ of the peptide may possibly be adsorbed and subsequently eluted from the MOF after two incubations, except for MIL-100(Fe) where $\mathrm{A} \beta$ was not fully adsorbed. When the MOFs were treated with an aqueous solution containing $25 \%$ acetonitrile and $0.1 \%$ trifluoroacetic acid, several solutions contained desorbed $\mathrm{A} \beta$ peptide when probed

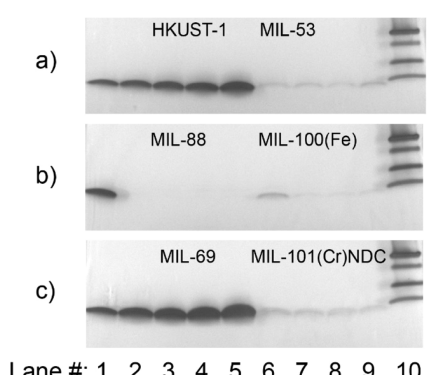

Figure 2. Desorption results measured by gel electrophoresis and silver staining are shown. On each gel, lane 1 is the control/initial (from one of the two incubations), lane 10 is a reference ladder (with bands at $10,15,20$, and $25 \mathrm{kD}$ shown), and lanes $2-5$ and $6-9$ are quadruplicate samples with each MOF. (a) HKUST-1 and MIL-53; (b) MIL-88B and MIL-100(Fe), and (c) MIL-69 and MIL-101(Cr) NDC.

with gel electrophoresis (Figure 2). HKUST-1 and MIL-69 eluted the highest amounts of peptide, with 160 and 150\%, respectively, relative to the single-incubation control (Figure 2a,c, lanes 2-5). The other MOFs retained the peptide to a greater extent, with only MIL-53 and MIL-101(Cr) NDC eluting more than $10 \%$ of peptide (about 13 and $17 \%$ of a single-incubation control, Figure 2a,c, lanes 6-9, respectively). UiO-66 and MIL-88B released $2 \%$ or less of adsorbed peptide according to gel electrophoresis. Increasing the acetonitrile concentration to $50 \%$ eluted a small additional amount of peptide from those that already had the greatest amount of desorbed peptide, HKUST-1, MIL-69, and MIL-101(Cr) NDC (about 15\% additional peptide), but did not change the overall trends of desorption. UiO-66 and MIL-88B which had shown limited desorption by gel electrophoresis were also exposed to hexafluoroisopropanol (HFIP) to test for additional desorption. This did not show significant additional elution of peptide from the MOFs. Analysis by SEM and powder X-ray diffraction of MOFs tested suggests that the MOFs themselves are not degraded during the adsorption and desorption processes (Figures S1 and S4).

After testing whether MOFs could adsorb and desorb $\mathrm{A} \beta$, the rate of adsorption for several MOFs was tested. MIL101(Cr) NDC showed particularly rapid adsorption compared to others tested. After $2 \mathrm{~min}$, less than $1 \%$ of the peptide remained in solution, as determined by silver staining. Fluorimetric analysis supports this, with the $2 \mathrm{~min}$ time point already below detection limits of the assay. UiO-66 and MIL-88B also showed rapid adsorption; by $10 \mathrm{~min}$ of incubation, peptide levels were below detection limits of both assays (Figure 3). MIL-53 was similar, showing 31\% peptide remaining after $2 \mathrm{~min}$ and complete adsorption by 10 min. Overall, for those tested in timed studies, data show that MIL-100(Fe) has the slowest rate of adsorption. When exposed to a full aliquot of $\mathrm{A} \beta$, we observe a slow, steady adsorption over the course of $2 \mathrm{~h}$, eventually nearing complete adsorption of a first aliquot, as seen in earlier studies (Figures $1 \mathrm{~b}$ and 3 ).

MIL-69 and MIL-101(Cr) NDC were tested against additional aliquots of $\mathrm{A} \beta$. Both are formed using the 2,6naphthalenedicarboxylic acid ligand and showed full adsorption of an initial aliquot but different apparent strengths of retention in desorption studies. In these experiments, samples were rinsed, dried, and then reincubated with another aliquot of dissolved $\mathrm{A} \beta$. In the case of MIL-101(Cr) NDC, subsequent 


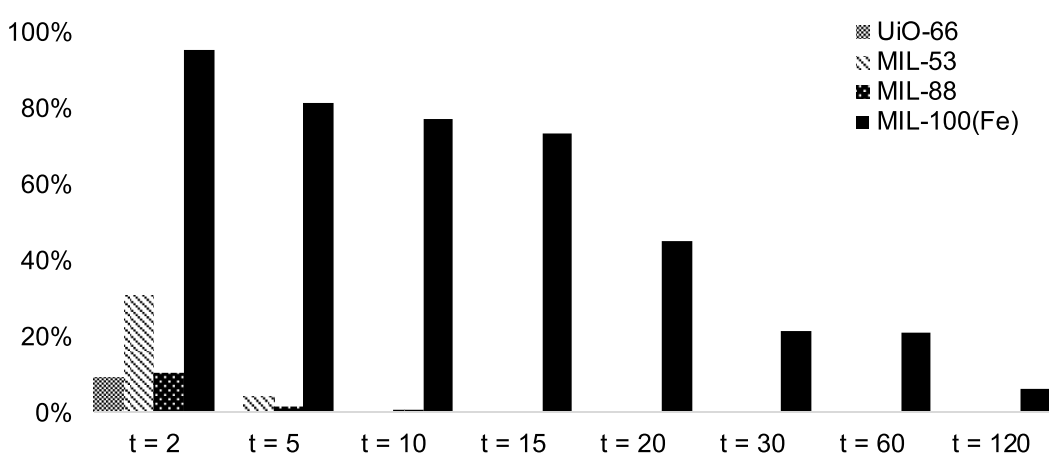

Figure 3. Examples of time-based incubations. Percentages are relative to the initial control concentration. Time values are in minutes. MIL$100(\mathrm{Fe})$ exhibits the slowest adsorption, while other MOFs adsorb most $\mathrm{A} \beta$ within 10 min.

incubations showed similar results to the first. Samples taken after 2 and 5 min showed slightly more peptides present than at the same time points in the first incubation, but by $8 \mathrm{~min}$, little to no $\mathrm{A} \beta$ remained in solution. After serial incubation with up to five aliquots, the supernatant contained less than $1 \%$ of the original $\mathrm{A} \beta$ after just $15 \mathrm{~min}$. This can be compared to MIL-69, samples of which were treated with subsequent $1 \mathrm{~h}$ long incubations. With each subsequent incubation, the relative amount of $\mathrm{A} \beta$ remaining in the supernatant increased (full adsorption of the first incubation, with steadily decreasing adsorption until $\sim 65 \%$ of the control peptide level remained in solution for the 6th incubation), suggesting that the MOF is becoming saturated in its ability to adsorb the peptide, at least for the timeframe of these incubations. Overall, this suggests that MIL-101(Cr) NDC has a much higher adsorption capacity than MIL-69. Studies with insulin adsorption in our lab have observed the same trend, with adsorption by MIL-69 dropping off significantly in additional incubations, while MIL101(Cr) NDC showed sustained adsorption behavior. Given the larger window and pore dimensions in MIL-101(Cr) NDC, these trends are perhaps not surprising.

\section{DISCUSSION}

Overall, our experiments show that of the MOFs studied, most of them readily adsorb small amounts of $\mathrm{A} \beta$ within the course of an hour or less. Only MIL-100(Fe) showed slower adsorption behavior, taking up to $2 \mathrm{~h}$ to achieve near-complete adsorption of the peptide in solution. Based on visualization by SEM, particle size does not appear to account for the slow MIL-100(Fe) rate (Figures S1 and S2 and Table S1). Adsorption rates might provide clues to the adsorption mechanism, especially for those observed to happen similarly quickly (within the first $10 \mathrm{~min}$ ). This might suggest that these MOFs follow similar mechanisms. Perhaps MIL-100(Fe), with its slower adsorption and low elution, adsorbs more $\mathrm{A} \beta$ inside the MOF pores, not just on the surface. ${ }^{3 b}$ Our results suggest that the peptide is adsorbed rather than being hydrolyzed by the MOF because the intact peptide can be desorbed from several MOFs after incubation and fluorimetric analysis would likely show detectable concentrations of small peptides in the samples if $\mathrm{A} \beta$ were hydrolyzed, but this is not observed. Additional work in our lab with adsorption of stained protein ladder components also support this general conclusion, as the dyed proteins either remain in solution in their intact size or are adsorbed by the MOFs. No new bands corresponding to cleaved proteins are observed. The desorption results also suggest that $\mathrm{A} \beta$ is not simply aggregating and collecting with the MOF solids, as acetonitrile has been shown to increase aggregation rates, so any peptide detected was likely desorbed from the MOFs and not the result of disrupted aggregates.? Supernatant solutions of several MOFs with strong retention were also probed with thioflavin $\mathrm{T}$ both after incubation and desorption. No increase in fluorescence was observed, again suggesting that $\mathrm{A} \beta$ has not aggregated. ${ }^{8}$

While uptake appears to be similar based on observed rates, other than MIL-100(Fe), elution experiments suggest that differences exist in interaction strengths between the MOFs and $\mathrm{A} \beta$. Some retain most or all adsorbed peptide, even after treatment with $50 \%$ acetonitrile, while others such as HKUST1 and MIL-69 readily desorb most of the adsorbed peptide. These differences could result from how the peptides are adsorbed. In the cases where only a small amount of peptide was eluted, perhaps the $\mathrm{A} \beta$ eluted was only adsorbed to the surface of the powders, compared to stronger interactions retaining most of the remaining peptides inside the pores. In the cases of HKUST-1 and MIL-69, if peptide is adsorbed in the pores, the interactions are weaker and more readily disrupted by the aqueous acetonitrile solutions, whereas UiO66, MIL-53, and MIL-88B feature interactions that are not disrupted by the desorption solutions. Ligand or metal identity alone does not explain the retention trends observed. For example, MIL-69 and MIL-53 both feature aluminum ions but show vastly different retention, as do HKUST-1 and MIL$100(\mathrm{Fe})$ which both feature trimesic acid linkers. The MIL-69 synthesized has been reported to have limited porosity, ${ }^{5 \mathrm{c}}$ so surface adsorption that is strong enough to resist elution with water but is disrupted with the water/acetonitrile solution is likely in this case. Both MIL-88B and MIL-53 have been shown to have structural flexibility and exhibit a "breathing effect", and they may be capable of adsorbing and trapping the peptide, as has been observed for the same MOFs when adsorbing ibuprofen.

In terms of saturation, how much peptide could a MOF possibly hold? Considering that MIL-101(Cr) NDC appears to have a high capacity for $\mathrm{A} \beta$ adsorption compared to other MOFs tested in these studies, we looked at this case in more detail. The estimated pore volume for MIL-101(Cr) NDC has been reported to be $2.35 \mathrm{~cm}^{3} / \mathrm{g}$. ${ }^{5 \mathrm{f}} \mathrm{A} \beta(1-40)$ has an estimated volume of $\sim 5200 \AA^{3}$, meaning that approximately $3 \mathrm{mg}$ peptide/mg MOF if we assume $100 \%$ filling. ${ }^{10}$ In supramolecular host-guest interactions, the maximum guest:host volume ratio is often approximately 0.6 , giving about $1.8 \mathrm{mg}$ of potential peptide adsorption. ${ }^{11}$ Others have measured adsorption of lipase enzyme by covalent-organic frameworks in the range of $1 \mathrm{mg} / \mathrm{mg}$, suggesting that these values are reasonable as maximums. ${ }^{12}$ However, it is probably unlikely 
that all the pore space would be occupied by the peptide as it may be difficult for peptide to fully penetrate the MOF interior, the peptide might be too large to fit in one pore alone, and solvent competition likely exists as well because of the large concentration differences between peptide and solvent. It is not surprising then that we have observed saturation behavior of several of the MOFs with far smaller masses of peptide.

\section{CONCLUSIONS}

We have shown that seven different MOFs readily adsorb small masses of $\mathrm{A} \beta$, with different rates of adsorption and, more notably, different strengths of retention for adsorbed peptide. This expands the number of MOFs known to interact with $\mathrm{A} \beta$ quite significantly. Based on these results, in situations where it is desired that peptide be temporarily sequestered, then released, HKUST-1 and MIL-69 are good options. UiO-66, MIL-88B, and MIL-53 combine strong adsorption with nearcomplete retention, a combination of potential use for removal of $\mathrm{A} \beta$ peptide from a solution to lower the concentration or for additional structural studies. The MOFs also adsorb different masses of $\mathrm{A} \beta$ in saturation studies. MIL-101(Cr) NDC has the largest reported pore windows and dimensions of the MOFs tested. Compared to MIL-69, which also uses the 2,6naphthalenedicarboxylic acid ligand, MIL-101(Cr) NDC exhibits faster and higher capacity adsorption, with stronger retention of the peptide as well. Further studies could more thoroughly investigate the adsorption mechanism and interactions between peptide and MOF surfaces or use the MOFs as a platform for structural studies.

\section{MATERIALS AND METHODS}

MOF Synthesis. We studied seven MOFs, which were selected because they appeared to be generally water-stable or had been previously shown to interact with proteins and/or peptides according to other reports. HKUST-1, UiO-66, MIL53, MIL-69, MIL-88B, MIL-100(Fe), and MIL-101(Cr) NDC were synthesized according to literature procedures and characterized by powder X-ray diffraction and ATR-IR. Unless additional purification treatments were prescribed, the isolated solids were used as synthesized. ${ }^{5}$ Precursor materials were obtained from commercial sources and used without additional purification. Ligands used were terephthalic (MIL-53, MIL$88 \mathrm{~B}$, UiO-66), trimesic (MIL-100(Fe) and HKUST-1), and 2,6-naphthalenedicarboxylic acid (MIL-69 and MIL-101(Cr) NDC). Metal nitrates or chlorides were used as metal ion sources, incorporating $\mathrm{Al}^{3+}$ (MIL-53 and MIL-69), $\mathrm{Fe}^{3+}$ (MIL$88 \mathrm{~B}$ and MIL-100(Fe)), $\mathrm{Cr}^{3+}$ (MIL-101(Cr) NDC), $\mathrm{Cu}^{2+}$ (HKUST-1), and $\mathrm{Zr}^{4+}$ (UiO-66).

Peptide Preparation. Synthetic A $\beta(1-40)$ was purchased as lyophilized peptide (Rockland Immunochemical) and is reported as greater than $95 \%$ specific peptide. Solid peptide was dissolved in HFIP with sonication and vortex treatments until no solids or cloudiness were observed (sometimes taking up to several hours), divided into aliquots (generally $50 \mu \mathrm{L}$ / aliquot), and evaporated overnight in a fumehood, producing a clear film on the bottom of the low-retention microcentrifuge tubes. ${ }^{6}$ In most studies, an aliquot of HFIP-treated A $\beta(1-40)$ was dissolved first in $25 \mu \mathrm{L}$ of DMSO, and then, $225 \mu \mathrm{L}$ of water was added, with $3 \times 1 \mathrm{~min}$ alternating sonication and vortex treatments after each solvent was added, producing a solution with $\mathrm{pH}$ near 6 . The target peptide concentration was
$200 \mu \mathrm{g} / \mathrm{mL}$, although measured initial concentrations using fluorimetry were lower, in the range of $40-70 \mu \mathrm{g} / \mathrm{mL}$, likely due to limited solubility of $\mathrm{A} \beta$. After dissolving, the solutions were transferred to new microfuge tubes to avoid concentration changes because of additional $\mathrm{A} \beta$ dissolving during the experiment.

Adsorption. Both fixed, single-point and timed, multiplepoint studies were conducted. Peptide solutions (in whole or as aliquots) were transferred to microcentrifuge tubes containing massed MOF powders (around $5 \mathrm{mg}$ ). After agitation to fully suspend the MOF powders, the samples were allowed to incubate, generally for $2 \mathrm{~h}$ in a full-length, singlepoint incubation. The samples were kept at $4{ }^{\circ} \mathrm{C}$ during the incubation and agitated with three resuspensions per hour to ensure adequate mixing. The samples were then centrifuged for $3 \mathrm{~min}$ at 10,000 rpm, and supernatants were collected. Before second incubations, the MOF solids were rinsed with $\mathrm{H}_{2} \mathrm{O}$, dried in a fumehood overnight, and then re-incubated with new $\mathrm{A} \beta$ peptide solutions in the same manner described above. The $\mathrm{H}_{2} \mathrm{O}$ rinse solutions showed no significant presence of $\mathrm{A} \beta$, suggesting that water alone does not elute or wash off the peptide from the MOF.

For time-based, multi-point studies, larger volumes were added to the MOF solids, and then, $10 \mu \mathrm{L}$ aliquots were taken and immediately stored in the freezer to minimize aggregation at $2,5,10,15,20,30,60$, and $120 \mathrm{~min}$ for several MOFs (UiO66, MIL-53, MIL-88B, MIL-100(Fe), and MIL-101(Cr) NDC). At each time point, the samples were centrifuged, aliquots were taken, and then solids were resuspended (timing was paused during these steps).

Quantification. Peptide content was compared by gel electrophoresis with visualization by silver staining and/or quantified using a Qubit 3.0 fluorometer or BCA concentration assays. ${ }^{13}$ Dilutions of stock solutions showed a linear relationship, supporting the general reliability of the Qubit for quantification of $\mathrm{A} \beta$ concentrations within detection limits. Peptide content in samples was also compared by gel electrophoresis and silver staining with the band densities measured using ImageJ, setting the control as $100 \%$. Gel electrophoresis was performed using Bio-Rad 4-20\% MiniPROTEAN gels with Bio-Rad Precision Plus standards.

Desorption. Desorption of proteins and peptides from MOFs has been achieved by others through treatment with aqueous solutions containing acetonitrile. ${ }^{3 \mathrm{c}}$ We exposed samples to solutions with either $25 \%$ or $50 \%$ acetonitrile and $0.1 \%$ trifluoroacetic acid. MOFs that showed limited or no desorption by this method were also treated with HFIP to test for desorption with this solvent. Dried solid MOFs were treated with desorption solutions for $1 \mathrm{~h}$ with several resuspensions and then centrifuged for $3 \mathrm{~min}$ at 10,000 rpm, and the supernatant was collected.

\section{ASSOCIATED CONTENT}

\section{Supporting Information}

The Supporting Information is available free of charge at https://pubs.acs.org/doi/10.1021/acsomega.0c04019.

Characterization data from scanning electron microscopy and powder X-ray diffraction and literature values for structural properties of MOFs utilized (PDF) 


\section{AUTHOR INFORMATION}

\section{Corresponding Author}

Zachary L. Mensinger - Department of Natural Sciences, College of Sciences, Metropolitan State University, St. Paul, Minnesota 55106, United States; 이이.org/0000-00016664-7286; Phone: 651-793-1497;

Email: zack.mensinger@metrostate.edu

\section{Authors}

Brenna L. Cook - Division of Science and Math, University of Minnesota-Morris, Morris, Minnesota 56267, United States

Elsie L. Wilson - Division of Science and Math, University of Minnesota-Morris, Morris, Minnesota 56267, United States

Complete contact information is available at:

https://pubs.acs.org/10.1021/acsomega.0c04019

\section{Author Contributions}

The manuscript was written by Z.L.M., with experimental contributions by all authors. All authors have given approval to the final version of the manuscript.

\section{Notes}

The authors declare no competing financial interest.

\section{ACKNOWLEDGMENTS}

Financial support for this research was provided by Metropolitan State University College of Sciences; University of Minnesota, Morris (UMM) Faculty Research Enhancement Funds supported by the University of Minnesota Office of the Vice President for Research; the UMM Division of Science and Mathematics; and Grant-in-Aid of Research, Artistry and Scholarship from the Office of the Dean of the University of Minnesota Graduate School (Proposal \#23122). This work was also supported in part by a grant to the University of Minnesota, Morris from the Howard Hughes Medical Institute through the Precollege and Undergraduate Science Education Program. Z.L.M. also personally thanks all of his previous research mentors, who have provided invaluable guidance throughout his scientific education.

\section{REFERENCES}

(1) (a) Findeis, M. A. The role of amyloid $\beta$ peptide 42 in Alzheimer's disease. Pharmacol. Ther. 2007, 116, 266-286. (b) Murphy, M. P.; LeVine, H. Alzheimer's Disease and the $\beta$ Amyloid Peptide. J. Alzheim. Dis. 2010, 19, 311. (c) Finder, V. H.; Glockshuber, R. Amyloid-beta aggregation. Neurodegener. Dis. 2007, 4, 13-27. (d) Stöhr, J.; Watts, J. C.; Mensinger, Z. L.; Oehler, A.; Grillo, S. K.; DeArmond, S. J.; Prusiner, S. B.; Giles, K. Purified and synthetic Alzheimer's amyloid beta $(\mathrm{A} \beta)$ prions. Proc. Natl. Acad. Sci. U.S.A. 2012, 109, 11025-11030.

(2) (a) Horcajada, P.; Gref, R.; Baati, T.; Allan, P. K.; Maurin, G.; Couvreur, P.; Férey, G.; Morris, R. E.; Serre, C. Metal-Organic Frameworks in Biomedicine. Chem. Rev. 2012, 112, 1232-1268. (b) Sumida, K.; Rogow, D. L.; Mason, J. A.; McDonald, T. M.; Bloch, E. D.; Herm, Z. R.; Bae, T.-H.; Long, J. R. Carbon Dioxide Capture in Metal-Organic Frameworks. Chem. Rev. 2012, 112, 724-781. (c) Stock, N.; Biswas, S. Synthesis of Metal-Organic Frameworks (MOFs): Routes to Various MOF Topologies, Morphologies, and Composites. Chem. Rev. 2012, 112, 933-969.

(3) (a) Chen, Y.; Han, S.; Li, X.; Zhang, Z.; Ma, S. Why Does Enzyme Not Leach from Metal-Organic Frameworks (MOFs)? Unveiling the Interactions between an Enzyme Molecule and a MOF. Inorg. Chem. 2014, 53, 10006-10008. (b) Chen, Y.; Lykourinou, V.; Vetromile, C.; Hoang, T.; Ming, L.-J.; Larsen, R. W.; Ma, S. How Can Proteins Enter the Interior of a MOF? Investigation of Cytochrome $\mathrm{c}$
Translocation into a MOF Consisting of Mesoporous Cages with Microporous Windows. J. Am. Chem. Soc. 2012, 134, 13188-13191. (c) Gu, Z.-Y.; Chen, Y.-J.; Jiang, J.-Q.; Yan, X.-P. Metal-organic frameworks for efficient enrichment of peptides with simultaneous exclusion of proteins from complex biological samples. Chem. Commun. 2011, 47, 4787-4789. (d) Lykourinou, V.; Chen, Y.; Wang, X.-S.; Meng, L.; Hoang, T.; Ming, L.-J.; Musselman, R. L.; Ma, $\mathrm{S}$. Immobilization of MP-11 into a Mesoporous Metal-Organic Framework, MP-11@mesoMOF: A New Platform for Enzymatic Catalysis. J. Am. Chem. Soc. 2011, 133, 10382-10385. (e) Kenneth, J. B.; Thomas, J. P.; Rita, H., Microperoxidase-11 Immobilized in a Metal Organic Framework. Biomolecular Catalysis; American Chemical Society, 2008; Vol. 986, pp 76-98.

(4) (a) Wang, J.; Fan, Y.; Tan, Y.; Zhao, X.; Zhang, Y.; Cheng, C.; Yang, M. Porphyrinic Metal-Organic Framework PCN-224 Nanoparticles for Near-Infrared-Induced Attenuation of Aggregation and Neurotoxicity of Alzheimer's Amyloid- $\beta$ Peptide. ACS Appl. Mater. Interfaces 2018, 10, 36615-36621. (b) Yu, D.; Guan, Y.; Bai, F.; Du, Z.; Gao, N.; Ren, J.; Qu, X. Metal-Organic Frameworks Harness Cu Chelating and Photooxidation Against Amyloid $\beta$ Aggregation in Vivo. Chemistry 2019, 25, 3489-3495. (c) Zhao, G.; Wang, Y.; Li, X.; Yue, Q.; Dong, X.; Du, B.; Cao, W.; Wei, Q. Dual-Quenching Electrochemiluminescence Strategy Based on Three-Dimensional Metal-Organic Frameworks for Ultrasensitive Detection of AmyloidB. Anal. Chem. 2019, 91, 1989-1996. (d) Wang, Y.; Zhang, Y.; Sha, H.; Xiong, X.; Jia, N. Design and Biosensing of a Ratiometric Electrochemiluminescence Resonance Energy Transfer Aptasensor between a g- $\mathrm{C}_{3} \mathrm{~N}_{4}$ Nanosheet and $\mathrm{Ru} @ M O F$ for Amyloid- $\beta$ Protein. ACS Appl. Mater. Interfaces 2019, 11, 36299-36306. (e) Han, J.; Zhang, M.; Chen, G.; Zhang, Y.; Wei, Q.; Zhuo, Y.; Xie, G.; Yuan, R.; Chen, $\mathrm{S}$. Ferrocene covalently confined in porous MOF as signal tag for highly sensitive electrochemical immunoassay of amyloid- $\beta$. J. Mater. Chem. B 2017, 5, 8330-8336. (f) Wang, X. Z.; Du, J.; Xiao, N. N.; Zhang, Y.; Fei, L.; LaCoste, J. D.; Huang, Z.; Wang, Q.; Wang, X. R.; Ding, B. Driving force to detect Alzheimer's disease biomarkers: application of a thioflavine T@Er-MOF ratiometric fluorescent sensor for smart detection of presenilin 1, amyloid $\beta$-protein and acetylcholine. Analyst 2020, 145, 4646-4663.

(5) (a) Horcajada, P.; Salles, F.; Wuttke, S.; Devic, T.; Heurtaux, D.; Maurin, G.; Vimont, A.; Daturi, M.; David, O.; Magnier, E.; Stock, N.; Filinchuk, Y.; Popov, D.; Riekel, C.; Férey, G.; Serre, C. How Linker's Modification Controls Swelling Properties of Highly Flexible Iron(III) Dicarboxylates MIL-88. J. Am. Chem. Soc. 2011, 133, 17839-17847. (b) Katz, M. J.; Brown, Z. J.; Colón, Y. J.; Siu, P. W.; Scheidt, K. A.; Snurr, R. Q.; Hupp, J. T.; Farha, O. K. A facile synthesis of UiO-66, UiO-67 and their derivatives. Chem. Commun. 2013, 49, 9449-9451. (c) Loiseau, T.; Mellot-Draznieks, C.; Muguerra, H.; Férey, G.; Haouas, M.; Taulelle, F. Hydrothermal synthesis and crystal structure of a new three-dimensional aluminumorganic framework MIL-69 with 2,6-naphthalenedicarboxylate (ndc), $\mathrm{Al}(\mathrm{OH})(\mathrm{ndc}) \cdot \mathrm{H} 2 \mathrm{O}$. Compt. Rendus Chem. 2005, 8, 765-772. (d) Seo, Y.-K.; Yoon, J. W.; Lee, J. S.; Lee, U.-H.; Hwang, Y. K.; Jun, C.-H.; Horcajada, P.; Serre, C.; Chang, J.-S. Large scale fluorinefree synthesis of hierarchically porous iron(III) trimesate MIL$100(\mathrm{Fe})$ with a zeolite MTN topology. Microporous Mesoporous Mater. 2012, 157, 137-145. (e) Loiseau, T.; Serre, C.; Huguenard, C.; Fink, G.; Taulelle, F.; Henry, M.; Bataille, T.; Férey, G. A Rationale for the Large Breathing of the Porous Aluminum Terephthalate (MIL-53) Upon Hydration. Chem.-Eur. J. 2004, 10, 1373-1382. (f) Sonnauer, A.; Hoffmann, F.; Fröba, M.; Kienle, L.; Duppel, V.; Thommes, M.; Serre, C.; Férey, G.; Stock, N. Giant Pores in a Chromium 2,6Naphthalenedicarboxylate Open-Framework Structure with MIL-101 Topology. Angew. Chem., Int. Ed. 2009, 48, 3791-3794. (g) Li, B.; Chen, D.; Wang, J.; Yan, Z.; Jiang, L.; Deliang, D.; He, J.; Luo, Z.; Zhang, J.; Yuan, F. MOFzyme: Intrinsic protease-like activity of $\mathrm{Cu}-$ MOF. Sci. Rep. 2014, 4, 6759.

(6) Lambert, M. P.; Viola, K. L.; Chromy, B. A.; Chang, L.; Morgan, T. E.; Yu, J.; Venton, D. L.; Krafft, G. A.; Finch, C. E.; Klein, W. L. 
Vaccination with soluble $\mathrm{A} \beta$ oligomers generates toxicity-neutralizing antibodies. J. Neurochem. 2001, 79, 595-605.

(7) Shen, C. L.; Murphy, R. M. Solvent Effects on Self-Assembly of $\beta$-Amyloid Peptide. Biophys. J. 1995, 69, 640-651.

(8) Xue, C.; Lin, T. Y.; Chang, D.; Guo, Z. Thioflavin T as an amyloid dye: fibril quantification, optimal concentration and effect on aggregation. R. Soc. Open Sci. 2017, 4, 160696.

(9) Pham, H.; Ramos, K.; Sua, A.; Acuna, J.; Slowinska, K.; Nguyen, T.; Bui, A.; Weber, M. D. R.; Tian, F. Tuning Crystal Structures of Iron-Based Metal-Organic Frameworks for Drug Delivery Applications. ACS Omega 2020, 5, 3418-3427.

(10) Center for Biotechnology, Northwestern University. Peptide Properties Calculator. http://biotools.nubic.northwestern.edu/ proteincalc.html (accessed May 24, 2019).

(11) Mecozzi, S.; Rebek, J., Jr. The 55 \% Solution: A Formula for Molecular Recognition in the Liquid State. Chem.-Eur. J. 1998, 4, $1016-1022$.

(12) Sun, Q.; Fu, C.-W.; Aguila, B.; Perman, J.; Wang, S.; Huang, H.Y.; Xiao, F.-S.; Ma, S. Pore Environment Control and Enhanced Performance of Enzymes Infiltrated in Covalent Organic Frameworks. J. Am. Chem. Soc. 2018, 140, 984-992.

(13) Mantile, F.; Trovato, M.; Santoni, A.; Barba, P.; Ottonello, S.; De Berardinis, P.; Prisco, A. Alum and Squalene-Oil-in-Water Emulsion Enhance the Titer and Avidity of Anti-A $\beta$ Antibodies Induced by Multimeric Protein Antigen (1-11)E2, Preserving the Igg1-Skewed Isotype Distribution. PLoS One 2014, 9, No. e101474. 